Notfall Rettungsmed $2021 \cdot 24: 773-774$ https://doi.org/10.1007/s10049-021-00898-6 Angenommen: 27. April 2021

(c) Springer Medizin Verlag GmbH, ein Teil von Springer Nature 2021

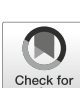

\author{
Michael Christ ${ }^{1} \cdot$ Marc-André Weber $^{2} \cdot$ Stefan Wirth $^{3}$ \\ ${ }^{1}$ Notfallzentrum, Luzerner Kantonsspital, Spitalstrasse, Luzern 16, Schweiz \\ ${ }^{2}$ Institut für Diagnostische und Interventionelle Radiologie, Kinder- und Neuroradiologie, \\ Universitätsmedizin Rostock, Rostock, Deutschland \\ ${ }^{3}$ Institut für Radiologie und Nuklearmedizin, Schwarzwald-Baar-Klinikum, Villingen-Schwenningen, \\ Deutschland
}

\title{
Radiologische Diagnostik in der Notfallmedizin
}

Computertomographie (CT) für die klinische Entscheidungsfindung sofort veranlasst und immer häufiger direkt in der Notaufnahme durchgeführt wird. Auch dies führt zu einem Anstieg der durchgeführten Untersuchungen verbunden mit einer Anpassung der internen Logistik.

Einige Zentren haben zwischenzeitlich notfallrelevante Diagnostik und in größeren Zentren auch die Akuttherapie logistisch und operativ in Notfallzentren integriert (Schockraum-CT, zerebrale Perfusionsbildgebung, „trauma bays“, Interventionsversorgung für akute Blutungen und Infarkte aller Körperregionen). In angloamerikanischen und europäischen Ländern entwickelt sich die Subspezialisierung der Notfallradiologie. Diese ist heute in den USA ein wichtiger Schritt zur Durchsatzsteigerung in Bezug auf Notfallpatienten (siehe https://www. healthimaging.com/topics/healthcareeconomics/emergency-radiologyemergency-medicine). Auch in Europa findet diese Entwicklung nun immer mehr statt, wobei sich dies radiologisch meist auf die Diagnostik auswirkt, je nach Zentrum aber auch zunehmend die interventionelle Therapie in verschiedenem Umfang einschließt. Da Notfallradiologie und Notfallmedizin getrennte Fachgebiete sind, kann die Spanne der Zusammenarbeit dieser Disziplinen von erheblichen Barrieren bis hin zu einem gemeinsamen, vollständig integrierten Team reichen.

Ein wichtiges Thema in der Notfalldiagnostik sind Herausforderungen in der Kommunikation: Die angeordneten Untersuchungsaufträge sind nicht selten zu wenig gezielt. Dies sowie unterschiedliche Sichtweisen von Indikationsstellung und Umfang der Untersuchungen können zu längeren Diskussionen zwischen Radiologen und Notfallmedizinern führen. Manchmal ist dies sinnvoll und notwendig, meist bedeutet es aber eigentlich vermeidbaren Reibungsverlust. Hinzu kommt, dass die machbare Leistungsmenge durch Ressourcen limitiert ist. Auch deshalb ist es sinnvoll, Spielräume zu schaffen für besonders dringliche Notfälle, und dass nichtlebensbedrohliche Notfälle ressourcenschonend und ablauforientiert geplant werden. Langfristig sinnvoll wäre sicherlich eine Integration von Notfallradiologie und Notfallmedizin im Sinne einer besseren patientenzentrierten Ablaufstruktur. In Deutschland ist das große Feld der Notfallradiologie im Rahmen der fachärztlichen Weiterbildung integriert, stellt aber nicht wie etwa in Skandinavien, Spanien oder den USA ein eigenes Kompetenzgebiet im Sinne einer eigenen Fachvertretung oder weiterführenden Spezialisierung dar. Die Notfallradiologie spiegelt sich aktuell auch nicht in einer eigenen Arbeitsgemeinschaft der Deutschen Röntgengesellschaft wider. Auf europäischer Ebene existiert seit zehn Jahren die European Society of Emergency Radiology (ESER). Diese bietet eine curriculare Weiterbildung und eine Zertifizierung mit einem europäischen Diplom in Notfallradiologie (European Diploma in Emergency Radiology [EDER]) an, das von der European Society of Radiology (ESR) anerkannt ist. Aktuell gibt 
es unter anderem aus diesen Gründen Bestrebungen der engeren Kooperation zwischen Deutscher Röntgengesellschaft und ESER. Wir haben uns in dieser Ausgabe von Notfall+Rettungsmedizin das Ziel gesetzt, radiologisches Fachwissen, das für die Notfallversorgung essenziell ist, den Lesern unserer Zeitschrift vorzustellen und aktuelle Herausforderungen zu diskutieren.

Eingeleitet wird diese Ausgabe durch den Beitrag unserer chirurgischen Kollegen um G. Alsfasser zum Thema ,Was erwartet der Nichtradiologe vom Radiologen beim akuten Abdomen?“. Im Beitrag werden Definition und Vorstellungssymptome eines akuten Abdomens beschrieben. Die Autoren formulieren explizit, dass in der Situation einer Vier-Quadranten-Peritonitis auch auf eine radiologische Diagnostik verzichtet wird. Neben dem Bedürfnis, zeitnah vom Notfallradiologen die Befundung zu erhalten, wird betont, dass die Ursache der Beschwerden und die Lokalbefunde dargestellt werden, sodass das operative Vorgehen besser geplant werden kann.

\section{》) Die CT unter Reanimation führt beim leblosen Patienten zu einer erheblichen Beschleunigung der Diagnostik}

Im Beitrag von $A$. Gäble et al. wird es ein Update zur radiologischen Diagnostik bei Polytrauma und unter Reanimationsbedingungen geben. Die Autoren aus verschiedenen europäischen Institutionen stellen eine radiologische Polytraumaleitlinie vor, die unter anderem auch in das Weiterbildungscurriculum der ESER integriert ist. Interessant für den Nichtradiologen ist, dass verschiedene Untersuchungsprotokolle der CTDiagnostik, aber auch ein Stufenschema der Befundung vorgestellt und diskutiert werden. Sehr interessant sind des Weiteren die präsentierten Fallberichte einer CT-Untersuchung unter Reanimation. Diese führt beim leblosen Patienten zu einer erheblichen Beschleunigung der Diagnostik und hilft durch die häufige Ursachenfeststellung nicht nur, sofort die richtigen Maßnahmen einzuleiten, son- dern unterstützt im leider häufigen Fall immerhin dabei, eine Beendigung der CPR zu rechtfertigen.

Die Kolleginnen und Kollegen um A. Reichelt präsentieren die Modalitäten der Bildgebung bei Patienten mit plötzlichen Brust- bzw. Unterleibsschmerzen. Die Autoren präsentieren typische Krankheitsbilder und erklären die dafür notwendigen radiologischen Protokolle und Untersuchungen. Spannend in diesem Kontext ist der Abschnitt über die "Triple-rule-out-CT“ bei unklaren Thoraxschmerzen, die in vielen Zentren noch nicht routinemäßig etabliert ist. Die kritische Auseinandersetzung mit der längeren Untersuchungsdauer, höheren Strahlendosen und höheren Kontrastmittelmengen gibt möglicherweise eine Erklärung, warum diese Untersuchung zumindest in deutschsprachigen Zentren eher selten durchgeführt wird.

M. Armbruster und Kollegen stellen in ihrem Beitrag „Interventionelle Radiologie in der Notfallmedizin“ den immer wichtigeren gefäßinterventionellen, das heißt therapeutischen Teilbereich der Notfallradiologie vor. Sie beschreiben die verschiedenen von den Interventionalisten verwendeten Devices und Tools. Die arterielle Thrombektomie ist in vielen Zentren bei akutem ischämischem Schlaganfall „Standard“. Andere interventionelle Verfahren zur Blutstillung werden umfassend erklärt.

Abschließend folgt ein Beitrag von K.J. Wenger et al., in dem die Bedeutung schneller Magnetresonanzsequenzen zur Abklärung zerebraler Störungen ausführlich dargelegt wird. Dieser Beitrag ist etwas technisch orientiert und wird einige von uns Notfallmedizinern auch überfordern. Andererseitsillustriert er sehr gut, wie zentral die korrekte klinische Fragestellung für den Notfallradiologen ist, damit auch das richtige Verfahren und schnellste Protokoll für diese Fragestellung eingesetzt werden kann. Im akuten Setting ist die Magnetresonanztomographie aufwendig und erfordert zudem eine ausgesprochen hohe Patientenkooperation. Insofern sind schnellere Sequenzen ein Beitrag zu dem - derzeit bei Weitem nicht erreichbaren - Ziel, den hohen Informationsgehalt auch in der Akutversorgung mehr zu nutzen.
Wir hoffen, Ihnen, liebe Leserinnen und Leser, mit diesem Leitthema eine gute Zusammenfassung aktueller Themenfelder der Notfallradiologie zu präsentieren. Wir haben uns bemüht, mit einem „bunten Strauß“ repräsentative Bereiche aus den verschiedenen Modalitäten, Körperregionen und Versorgungsarten anzubieten. Unser besonderer Dank gilt auf das Allerherzlichste den Autoren für deren hervorragende Beiträge.

Wir wünschen Ihnen viel Spaß beim Lesen und freuen uns sehr, wenn Sie diese Zusammenstellung interessant und hilfreich für Ihre tägliche praktische Arbeit finden.

\section{Mit den besten Grüßen}

M. Christ

M.-A. Weber

S. Wirth

\section{Korrespondenzadresse}

Prof. Dr. Michael Christ

Notfallzentrum, Luzerner Kantonsspital, Spitalstrasse

6000 Luzern 16, Schweiz

michael.christ@luks.ch

Interessenkonflikt. M. Christ, M.-A. Weber und S. Wirth geben an, dass kein Interessenkonfliktbesteht. 